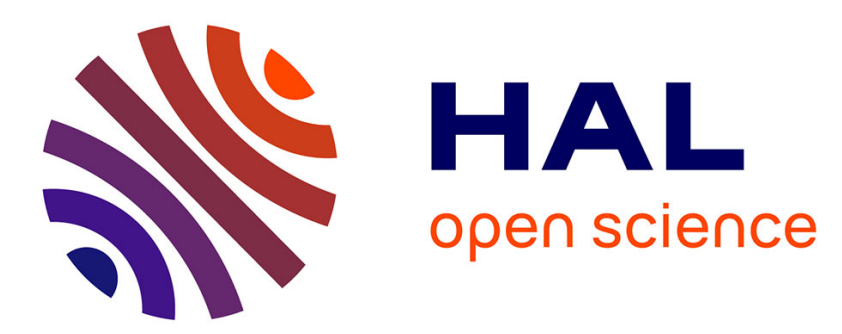

\title{
Articulation langue 1- langue 2 dans le répertoire langagier des élèves inscrits en programme immersif: quelles ressources lexicales pour les cours de sciences?
}

Sophie Babault, Michael Markey

\section{- To cite this version:}

Sophie Babault, Michael Markey. Articulation langue 1- langue 2 dans le répertoire langagier des élèves inscrits en programme immersif: quelles ressources lexicales pour les cours de sciences?. Canadian Modern Language Review / La revue canadian des langues vivantes, 2016, 72 (4), pp.454-479. 10.3138/cmlr.3364 . hal-02294926

\section{HAL Id: hal-02294926 \\ https://hal.science/hal-02294926}

Submitted on 24 Sep 2019

HAL is a multi-disciplinary open access archive for the deposit and dissemination of scientific research documents, whether they are published or not. The documents may come from teaching and research institutions in France or abroad, or from public or private research centers.
L'archive ouverte pluridisciplinaire HAL, est destinée au dépôt et à la diffusion de documents scientifiques de niveau recherche, publiés ou non, émanant des établissements d'enseignement et de recherche français ou étrangers, des laboratoires publics ou privés. 


\title{
Articulation langue 1- langue 2 dans le répertoire langagier des élèves inscrits en programme immersif : quelles ressources lexicales pour les cours de sciences?
}

\section{Sophie Babault et Michael Markey}

\begin{abstract}
Résumé : Cet article présente les résultats d'une étude visant à analyser chez des élèves inscrits en programme immersif les modalités d'articulation entre, d'une part, contenus lexicaux et contenus conceptuels et, d'autre part, L1, langue de communication familiale, et L2, langue de scolarisation. Après une mise au point sur l'état de la recherche à ce sujet, les résultats de l'analyse quantitative et qualitative sont présentés. L'expérimentation a été réalisée auprès de six classes d'élèves belges francophones de 5 e année d'école primaire. L'échantillon pris en compte est constitué de 54 élèves inscrits dans un programme immersif et de 50 élèves, constituant le groupe témoin, inscrits dans un programme classique d'une école de la même commune.
\end{abstract}

Mots clés : éducation bilingue, immersion, lexique, articulation L1-L2, articulation contenus lexicaux et contenus conceptuels, Belgique, français, néerlandais, cours de sciences

\begin{abstract}
This article presents the results of a study that analyzed, among students enrolled in an immersion programme, the linking processes between, on the one hand, lexical and conceptual contents and, on the other hand, the L1, the language spoken at home, and the L2, the schooling language. After a clarification of the status of current research on the subject, the results of the quantitative and qualitative analyses are presented. The experiment was performed in six classes of fifth-grade French-speaking Belgian students. The sample is made up of 54 students enrolled in an immersion programme and 50 students, the control group, enrolled in a regular programme, in a school located in the same district.
\end{abstract}

Keywords: bilingual education, immersion, lexicon, linking L1-L2, linking lexical and conceptual contents, Belgium, French, Dutch, science class 


\section{Introduction}

Si de nombreuses études ont été menées afin d'évaluer, de manière séparée, les compétences linguistiques des élèves dans leur langue 1 (désormais L1) ou dans la langue d'immersion (Stohler, 2006 ; Van de Craen, Surmont, Mondt et Ceuleers, 2012 ; etc.) nous disposons en revanche à ce jour de peu de données sur la façon dont s'articulent les compétences linguistiques bilingues autour des savoirs et des compétences disciplinaires développés en langue 2 (désormais L2). Il semble pourtant important de s'attacher à trouver des éléments de réponse à la question suivante : dans quelle mesure la compétence bilingue des élèves inscrits en parcours immersif s'applique-t-elle aux aspects linguistiques et discursifs relatifs aux disciplines enseignées en L2 ? Autrement dit, dans quelle mesure les élèves inscrits en immersion parviennent-ils à construire des ponts et à opérer des transferts entre, d'une part, leurs ressources linguistiques en L2, langue de construction de certains savoirs scolaires, et, d'autre part, leurs ressources en L1, langue de communication familiale et de verbalisation des expériences extrascolaires?

C'est à ces deux questions que nous avons tenté d'apporter des réponses en menant une recherche auprès d'élèves belges francophones scolarisés dans un programme d'immersion paritaire en néerlandais. Nous avons focalisé notre recherche sur les aspects lexicaux du répertoire langagier des élèves.

Après une mise au point sur les spécificités des compétences développées en contexte immersif, puis sur l'état de la recherche en matière d'articulation L1-L2 en contexte d'immersion ou, plus largement, de scolarisation en L2, nous présenterons les résultats de la recherche.

\section{Spécificités des compétences linguistiques développées en contexte immersif}

De très nombreuses dénominations sont utilisées pour désigner les programmes dans lesquels des élèves parlant la langue de la majorité linguistique sont scolarisés dans des classes où une autre langue est utilisée partiellement ou totalement comme médium d'instruction. Parmi ces dénominations, nous faisons le choix d'utiliser le terme programme immersif car c'est le terme officiellement employé par l'institution scolaire de la communauté française de Belgique pour désigner ce type de programme, lancé en 1998 et en expansion croissante depuis cette date.

Les programmes immersifs constituent une forme spécifique de développement d'un répertoire bi- ou plurilingue chez les élèves dans la mesure où, dans ces programmes, les compétences en L2 se 
développent parallèlement à la construction de savoirs et de compétences dans des disciplines dites non linguistiques (Gajo et Serra, 2000). De manière générale, en situation scolaire, que la langue de communication de la classe soit ou non la L1 des élèves, les éléments linguistiques utilisés dans le discours didactique ne se recoupent que de façon partielle avec ceux qu'on peut observer dans les discours extrascolaires. Cette spécificité est liée non seulement aux caractéristiques des domaines enseignés en classe, qui vont faire émerger du lexique nouveau destiné à désigner de nouveaux objets de connaissance, mais également au passage de la connaissance commune à la connaissance scientifique, qui implique différents niveaux de formulation (Vigner, 2001). Dans le cas d'une scolarisation en L1, c'est en s'appuyant sur un certain continuum entre la « langue » de l'école et celle de la maison que les enseignants aident leurs élèves à acquérir de manière conjointe les connaissances disciplinaires et les moyens linguistiques qui permettent de les intégrer dans des schémas cognitifs cohérents. Lorsque la construction de nouvelles connaissances passe par une L2, le recours à un continuum linguistique n'est pas toujours possible, ce qui a pour conséquence de modifier les modalités de construction de ces connaissances et du répertoire langagier qui les accompagne.

Une deuxième caractéristique du développement du répertoire bilingue des élèves en immersion vient du fait que les programmes immersifs entraînent pour la plupart de ces élèves une rupture linguistique nette entre l'espace scolaire, dans lequel la L2 occupe une place importante, et l'espace familial, dans lequel cette L2 est très peu présente, voire totalement absente, et parfois non maîtrisée par une partie ou la totalité des membres de la famille. Cette configuration a un impact sur la construction des répertoires langagiers, considérés comme « [embrassant] des compétences inégales, partielles, dans plusieurs langues dont la maîtrise changera, par ailleurs, en fonction des besoins toujours nouveaux le long d'une vie » (Lüdi, 1999, p. 47). Audelà des processus d'intégration de savoirs disciplinaires en L2, la rupture linguistique qui survient entre la sphère scolaire et la sphère extrascolaire de chaque élève est un point déterminant dans la construction des représentations sémantiques bilingues (de Groot, 1993). Cette rupture implique en effet que les processus de construction de la mémoire sémantique dépendent de divers réseaux de connexion entre les connaissances appréhendées à l'école en L2 et celles qui relèvent du savoir extrascolaire, véhiculé par la L1 de chaque élève, ce savoir extrascolaire étant lui-même inscrit dans un processus de construction à partir de formes de connaissance variées (expérience 
directe des élèves ou médiatisation par la lecture, la télévision, le discours d'autrui, etc.).

Les constituants lexicaux du répertoire langagier sont centraux dans le développement des compétences linguistiques en contexte d'immersion, car ils servent de point d'appui indispensable pour le développement conceptuel des apprenants. Au-delà de l'intérêt, dans une perspective actionnelle de didactique des langues, d'utiliser une L2 comme médium d'instruction pour d'autres disciplines, c'est probablement cette spécificité qui représente l'enjeu principal de l'enseignement en immersion : l'enseignement immersif renouvelle totalement l'approche de l'apprentissage des langues en mettant l'accent non seulement sur une compétence communicative globale, activable dans diverses situations d'interaction, mais également sur le rôle joué par les langues comme outils de conceptualisation (Robinson, 2001 ; Jaubert, 2007 ; etc.). L'appropriation d'une langue est nécessairement liée à des phénomènes de conceptualisation et de classification du réel. Dans les situations d'apprentissage " traditionnel » des langues étrangères, les démarches de classification du réel par le biais de la langue cible restent majoritairement limitées à des items courants, concrets pour la plupart, venant s'appuyer sur des termes connus des apprenants dans leur langue source, aux représentations sémantiques similaires ou en chevauchement partiel (exemples : maison, livre, rivière, repas, etc.). ${ }^{1}$ Il en va tout autrement dans le cas de l'enseignement immersif, pour lequel les apprenants sont confrontés à des termes et concepts qu'ils ne peuvent pas nécessairement relier à des représentations sémantiques déjà intériorisées (exemple en sciences : condensation, pression atmosphérique, oxygène, etc.). Les éléments lexicaux spécialisés font alors l'objet, chez les apprenants, d'une analyse visant à organiser le contenu sémantique puis, une fois intégrés, ils constitueront des bases pour les étapes suivantes de conceptualisation. Le lexique général joue également un rôle fondamental dans les démarches de conceptualisation en procurant aux apprenants des outils descriptifs et explicatifs précis qui favoriseront les processus de conceptualisation.

\section{Compétences linguistiques bilingues et compétences disciplinaires en contexte d'enseignement en L2 : état de la recherche}

La question du transfert de compétences d'une langue à l'autre chez les bilingues constitue depuis plusieurs décennies un point crucial parmi les chercheurs. S'appuyant sur plusieurs études menées durant les années 1970 auprès d'élèves scolarisés en programme 
bilingue, Cummins (1980) propose de distinguer, dans les compétences linguistiques des élèves, celles qui relèvent des compétences communicatives (qu'il présente sous l'acronyme BICS : basic interpersonal communication skills) et celles qui sont spécifiquement liées aux aspects cognitifs développés en situation scolaire (acronyme CALP : cognitive/academic language proficiency). L'intérêt majeur de cette distinction est qu'elle permet de mettre en lumière des éléments de compétence interdépendants d'une langue à l'autre : "These findings suggest that measures of the cognitive/academic aspects of L1 and L2 are assessing the same underlying dimension to a similar degree » (Cummins, 1980, p. 179). Cette théorie de l'interdépendance a été par la suite testée et relativisée dans d'autres contextes. Malakoff (1988) montre que, dans certaines conditions, l'immersion dans un programme monolingue en L2 aura des effets positifs à la fois sur les compétences en L1 et en L2. Verhoeven (1994), quant à lui, met en évidence un transfert très limité sur le plan lexical et syntaxique entre la langue d'instruction et la L1: "Thus, no clear evidence was found for interdependance from L1 to L2 lexical abilities » (p. 401). De manière générale, la non-prise en compte de facteurs sociaux semble constituer une limite de la théorie de Cummins (Genesee, 1984, Verhoeven, 1994).

Au-delà de l'évaluation des compétences en L1 ou L2, un certain nombre de recherches se sont focalisées ces dernières années sur les aspects discursifs de la construction de compétences chez les élèves scolarisés en parcours bilingue. Distinguant trois étapes successives dans l'histoire de la recherche sur l'enseignement bilingue, Gajo (2007) insiste ainsi sur la notion d'intégration qui caractérise la troisième étape : pour lui, il ne s'agit plus maintenant de vérifier l'impact de l'enseignement bilingue sur les compétences en L1 ou sur celles en L2, mais bien de mettre l'accent sur les modalités d'interrelation entre les compétences langagières et les savoirs disciplinaires, médiés par le discours : " Discourse is structured by both the subject and the linguistic paradigms, which are at the same time structured by it. Knowledge is shaped in this complex interrelation » (Gajo, 2007, p. 568). On retrouve de manière nette l'accent mis sur cette médiation opérée par le discours entre savoirs linguistiques et savoirs disciplinaires dans des recherches telles que celle de Alanen, Jäppinen et Nikula (2006), qui analysent les processus interactionnels de formation d'un concept géographique en L2 dans une classe d'élèves finlandais.

Le projet de recherche interdisciplinaire dirigé par Noyau au début des années 2000 apporte un autre éclairage à la question de l'articulation entre les compétences bilingues et les savoirs interdisciplinaires. Ce projet visait à analyser la « genèse du sujet bilingue francophone 
en situation diglossique en Afrique de l'ouest, à travers la scolarisation en français et l'expérience langagière dans l'environnement en langues premières » (Noyau, 2003, p. 1)Les différents travaux publiés dans le cadre de ce projet (Noyau, 2003, 2007 ; Noyau et Vellard, 2004, etc.), centrés sur des cours d'histoire, de sciences ou de mathématiques, présentent l'intérêt de mettre en lumière des spécificités discursives propres à chaque discipline, tout en intégrant à la réflexion une prise en compte des modalités de réalisation linguistique de tel ou tel aspect discursif dans les langues premières des élèves.

Enfin, il est utile de tenir compte des données sur l'articulation des compétences linguistiques et disciplinaires apportées par les chercheurs travaillant dans des contextes de scolarisation en L1. Ainsi que le note Jaubert (2007, p. 45) : «Se poser la question du lien entre langage et enseignement-apprentissage de savoirs scientifiques à l'école, c'est postuler que la relation didactique repose en partie sur des pratiques langagières mais surtout, que les savoirs scientifiques ont une dimension langagière irréductible qui ne saurait être totalement occultée au sein de l'institution scolaire ». Jaubert (2007) étudie ainsi, notamment, la place tenue par la négociation du sens dans les processus de conceptualisation : "La signification n'étant pas un donné, n'étant pas inhérente au vécu, mais un problème, c'est sa négociation qui se trouve être l'enjeu de la communication scolaire» (p. 94). La notion de concept est au centre d'un grand nombre de recherches prenant pour objet l'enseignement-apprentissage des sciences au niveau de l'école élémentaire (Astolfi, Peterfalvi et Vérin, 1998 ; Giordan et de Vecchi, 1994 ; Brossard et Fijalkow, 2002 ; Schneeberger et Vérin, 2009), reposant en partie sur les apports de Vygotski (1934/1985). Vygotski opère une distinction entre les " concepts spontanés ", relevant du savoir commun lié à la vie quotidienne de chaque individu, ayant un domaine de validité restreint, et les " concepts scientifiques » qui, eux, ont un espace de validité plus large, ont fait l'objet d'une négociation et d'une validation par la communauté scientifique et sont imbriqués au sein d'un système dans lequel ils sont reliés à un ensemble d'autres concepts. Les aspects communicatifs et linguistiques sont centraux dans les processus de construction, chez les élèves, d'un système de concepts scientifiques, à partir notamment du déjà-là que constituent les concepts spontanés. Vygotski insiste sur la durée de ces processus, liée au caractère " productif et non reproductif » des démarches opérées par les élèves : " A partir du moment où l'enfant apprend pour la première fois la signification d'un mot pour lui nouveau, le processus de développement du concept, loin de s'achever, ne fait que commencer» (Vygotski, 1934/1985, p. 214). 


\section{Présente recherche}

La présente recherche s'inscrit dans la lignée des travaux de Gajo, Noyau et des chercheurs cités ci-dessus qui ont travaillé sur les aspects linguistiques de l'apprentissage des sciences en L1. Nous nous démarquons cependant de ces différents travaux en faisant le choix de centrer notre démarche d'analyse sur le lexique comme outil de conceptualisation et de communication scientifique. Au-delà de problématiques concernant l'acquisition incidente du vocabulaire (Sylvén, 2010) ou les fréquences d'apparition lexicale (Nation, 2001), les études lexicales portant sur les contextes immersifs restent peu nombreuses. Nous souhaitons donc, par cette recherche, apporter un éclairage sur le lien qui unit lexique et construction conceptuelle en situation d'apprentissage en L2, ce qui pose inévitablement la question de l'articulation entre L1 et L2 dans le développement des ressources lexicales des élèves, en relation avec les deux niveaux de conceptualisation mis en lumière par Vygotski.

Après une démarche exploratoire prenant pour objet des cours d'histoire (Babault, 2008), nous avons publié une première étude centrée sur la problématique des cours de sciences (Babault et Markey, 2011) dans laquelle nous avons apporté de premiers résultats sur un petit échantillon d'élèves francophones de $5^{\mathrm{e}}$ année de l'école primaire, concernant les outils conceptuels et lexicaux développés par les élèves à partir d'une séquence pédagogique en néerlandais consacrée au thème des états de l'eau.

Dans la présente recherche, la démarche méthodologique suivie est en partie la même, mais l'échantillon est plus important et les données d'analyse sont élargies (mise en place d'un pré-test et comparaison avec un groupe témoin).

\section{Démarches de recueil et d'analyse des données}

La recherche a été menée auprès d'un échantillon de 54 élèves francophones de la province du Hainaut (Belgique) inscrits en $5^{\mathrm{e}}$ primaire dans un programme d'immersion paritaire (50\% des cours en français et $50 \%$ en néerlandais depuis la $1^{\mathrm{e}}$ primaire), complété par un groupe témoin de 50 élèves de $5^{\mathrm{e}}$ primaire suivant un cursus traditionnel en français dans la même commune. Après des observations réalisées à différents niveaux de scolarité, nous nous sommes fixés sur la $5^{\mathrm{e}}$ année de l'enseignement primaire, niveau dans lequel les élèves ont acquis des compétences suffisamment développées en néerlandais pour pouvoir s'appuyer réellement sur une médiation langagière. Nous avons cherché, pour les groupes témoins, des classes dont le 
profil socioculturel des élèves était comparable à celui des trois classes immersives. ${ }^{2}$

$\mathrm{Au}$ total, nous avons retenu six classes ${ }^{3}$ :

- Classes immersives : une classe de l'école des frères maristes (école confessionnelle, Mouscron), deux classes du Centre Educatif Européen (école communale, Mouscron)

- Groupes témoins : deux classes de l'école des frères maristes, une classe de l'école St Henri (école confessionnelle, Comines)

Dans un premier temps, une semaine environ avant l'expérimentation proprement dite, l'enseignant de chaque classe concernée a fait remplir aux élèves un prétest que nous avions élaboré dans le but de vérifier leurs connaissances disciplinaires et linguistiques sur le thème prévu pour la leçon servant de support à l'expérimentation. Puis, après avoir assisté à un cours de sciences en néerlandais dont nous avions préalablement défini le contenu avec l'enseignant, notre équipe $^{4}$ a réalisé deux entretiens individuels d'une dizaine de minutes chacun - l'un en français et l'autre en néerlandais - avec chaque élève des classes immersives. Pour chaque élève, les deux entretiens étaient espacés d'environ une heure. L'ordre de priorité du français ou du néerlandais était aléatoire.

En tenant compte à la fois de nos objectifs de recherche et des contraintes pédagogiques ou organisationnelles auxquelles les enseignants étaient soumis, nous avons fait le choix de focaliser la recherche sur une séquence pédagogique consacrée au thème de la pression de l'air. Nous avons fait un compte rendu précis du déroulement de la séance menée par le premier enseignant, puis nous avons demandé aux enseignants suivants de suivre autant que possible ce déroulement (y compris les exemples donnés) pour leur classe. Avec l'accord $\mathrm{du}$ premier enseignant, nous avons d'ailleurs fourni aux autres enseignants un support d'activité à distribuer aux élèves. Un compte rendu de chaque séance a été fait par les membres de notre équipe y ayant assisté, ce qui nous a permis de vérifier les éléments de similitude entre les démarches pédagogiques et les discours des différents enseignants. L'expérimentation a également été menée avec deux autres classes, tenues par un même enseignant pour la partie néerlandophone, mais nous avons finalement décidé ne pas inclure ces deux classes à l'échantillon total pris en compte dans cet article : trop de différences dans le contenu du cours rendaient peu opérationnelle la comparaison des productions des élèves.

La séance de cours s'appuyait sur une série de huit expériences réalisées par les élèves, qui remplissaient leur feuille d'activité en notant 
leurs observations, puis discutaient en grand groupe afin de mettre en évidence les principales caractéristiques de l'air. Les expériences étaient les suivantes :

1- une feuille de papier est posée en équilibre sur deux tas de livres. Les élèves soufflent sous la feuille.

2- les élèves mettent une boulette de papier dans le goulot d'une bouteille puis soufflent pour faire entrer la boulette dans la bouteille.

3- Une bougie est posée sur une assiette remplie de jus de fruits. On allume la bougie puis on pose un verre dessus.

4- On gonfle un ballon à l'intérieur d'une tasse puis on retire la tasse.

5- Une carte postale est posée sur un verre rempli à ras bord d'eau puis le verre est retourné.

6- On troue le fond d'une bouteille en plastique puis on la remplit d'eau, on ferme le bouchon et on met la bouteille verticalement.

7- Une balle de pingpong est placée au-dessus d'un sèche-cheveux.

8- On souffle entre deux balles de pingpong accrochées chacune à une corde et distantes de quelques centimètres.

Les entretiens commençaient par une tâche de rappel libre sur les expériences faites en classe. Ensuite, dans un deuxième temps, des questions plus précises, notamment sur le plan lexical et conceptuel, étaient posées à chaque élève. Quelques données concernant la biographie et les pratiques langagières des élèves étaient également recueillies en fin d'entretien.

Les entretiens ont été enregistrés, puis transcrits.

Parallèlement, la même démarche a été suivie avec les classes témoins, qui ont suivi le même contenu de cours, mais en français, et avec lesquelles des entretiens en français ont été réalisés.

Les classes immersives comprenaient quelques élèves ayant le néerlandais comme L1 et utilisant principalement le néerlandais en famille. Nous n'avons pas inclus ces élèves dans l'échantillon. La question de la prise en compte ou non dans l'échantillon s'est également posée pour les élèves utilisant à différents degrés de fréquence une autre langue que le français ou le néerlandais comme langue de communication familiale. Nous avons fait le choix d'inclure ces élèves dans l'échantillon à partir du moment où ils ont fait la totalité de leur scolarité maternelle et primaire en France ou en Belgique. Il était en effet irréaliste, à partir des seules pratiques déclarées, de chercher à poser une limite en matière de degrés d'utilisation des langues en famille. 
Après prise en compte de cet ensemble de critères, l'échantillon total se répartissait comme indiqué dans le Tableau 1 :

Tableau 1 : nombre d'élèves de l'échantillon

\begin{tabular}{lccr}
\hline & Classes immersives & Groupes témoins & total \\
\hline Classe 1 & 22 & & \\
Classe 2 & 15 & & \\
Classe 3 & 17 & 21 & \\
Classe 4 & & 17 & \\
Classe 5 & & 12 & \\
Classe 6 & & $\mathbf{5 0}$ & $\mathbf{1 0 4}$ \\
total & $\mathbf{5 4}$ & & \\
\hline
\end{tabular}

Une analyse quantitative et qualitative a été réalisée à partir de deux types de données pour les groupes immersifs et les groupes témoins :

- Pré-tests

- Entretiens en français

Dans le cadre de cet article, il nous a semblé utile de centrer notre analyse sur les entretiens en français en recherchant dans le discours des élèves des traces de leur construction de représentations lexico-notionnelle intégrées à leur L1. Dans certains cas, nous avons cependant éclairé les analyses qualitatives des productions individuelles par des données issues des entretiens menés en néerlandais avec les élèves concernés.

\section{Résultats : outils lexicaux et conceptuels appliqués à un cours de sciences}

\section{a) Savoirs lexico-conceptuels initiaux}

La leçon sur laquelle nous avons travaillé dans cet article était centrée autour de la pression de l'air.

Environ une semaine avant cette leçon, les élèves avaient complété individuellement un document visant à vérifier leurs savoirs lexicaux initiaux. Un premier test concernait des lexèmes généraux, tels que lucht (" air »), drukken (" pousser »), bewegen (" bouger »), stilstaan (" rester immobile »), dont la maîtrise nous semblait nécessaire à la compréhension de la leçon. ${ }^{5}$ Le test se présentait sous la forme suivante : chaque item était accompagné de quatre images parmi lesquelles les élèves devaient entourer celle qui correspondait à l'item, ainsi qu'on le voit dans la Figure 1 pour les items lucht et drukken: 
Articulation langue 1- langue 2 dans le répertoire langagier
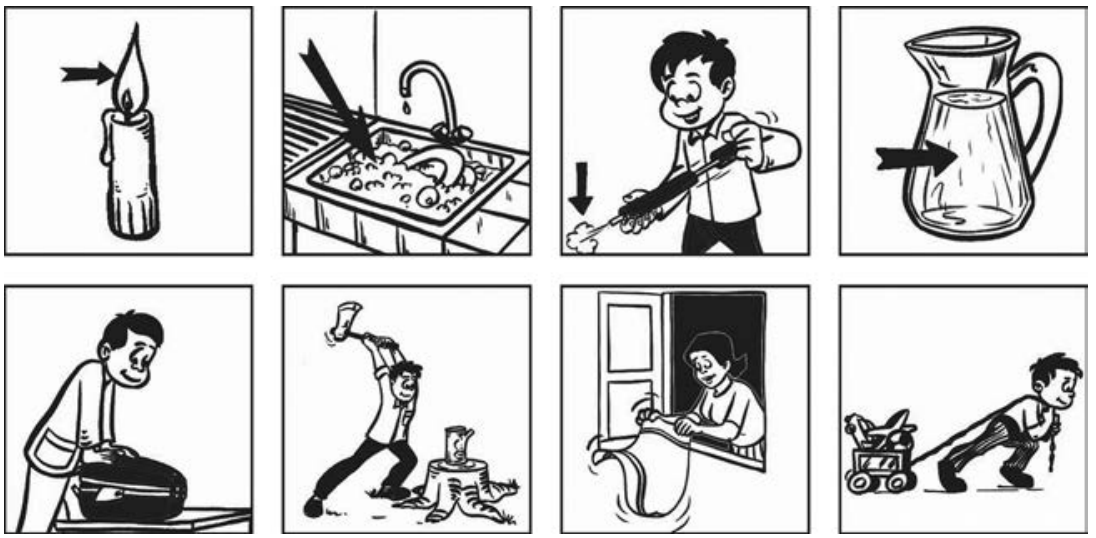

Figure 1 : Test lexical (lexèmes généraux)

lucht :

drukken:

Les scores obtenus par les élèves au premier test lexical sont repris dans la Figure 2 :

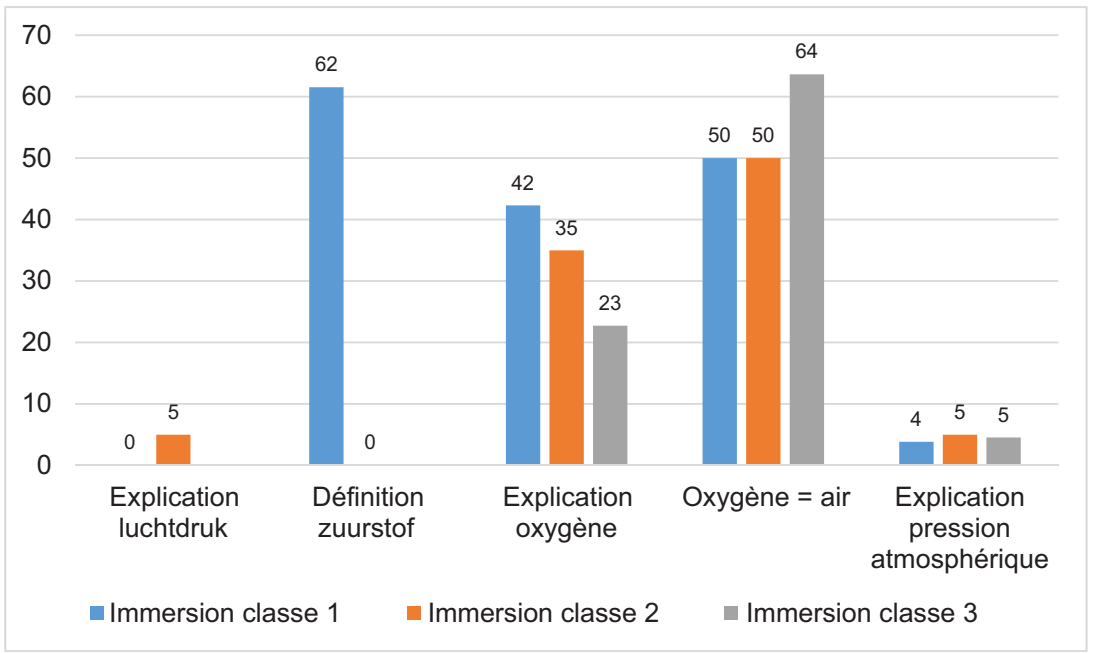

Figure 2 : Savoirs lexicaux initiaux 1 (groupes en immersion)

De manière générale, malgré des scores plus élevés pour la classe 2, ces résultats indiquent des tendances convergentes pour les élèves des trois classes : 
- le verbe stilstaan (« rester immobile») est globalement identifié par un pourcentage important d'élèves ;

- le verbe drukken (« pousser») est l'item le moins souvent identifié par les élèves des trois classes : de $40 \%$ pour la classe 2 à $4 \%$ pour la classe 1 ;

- le verbe bewegen («bouger») est identifié par environ deux tiers des élèves ;

- le nom lucht (« l'air») est identifié par environ la moitié des élèves.

Les équivalents français de ces quatre items ont été testés auprès des classes témoins à partir du même format de test (choix de l'image correspondant au terme présenté). Les pourcentages d'identification sont à $100 \%$ pour les trois classes témoins. Il nous semblait important, sur le plan méthodologique, d'effectuer cette vérification (ne serait-ce que pour vérifier la validité du test), mais il paraît clair que les quatre termes testés relèvent du lexique général maîtrisé par des enfants francophones d'une dizaine d'années.

Quant au deuxième test, il visait à évaluer la connaissance ou la non-connaissance d'unités lexicales reliées à des concepts centraux pour cette leçon : luchtdruk (" pression de l'air ») et zuurstof («oxygène »). Dans ce test, les unités lexicales étaient testées à la fois en néerlandais et en français. Le test se présentait sous la forme de quatre consignes concernant les unités lexicales en néerlandais :

- essaie de faire une phrase en néerlandais avec le mot luchtdruk;

- explique ce que veut dire le mot luchtdruk;

- essaie de faire une phrase en néerlandais avec le mot zuurstof;

- explique ce que veut dire le mot zuurstof.

Deux pages plus loin, quatre consignées étaient données à propos d'unités lexicales en français :

- Essaie de faire une phrase en français avec l'expression «pression atmosphérique »;

- Explique ce que veut dire l'expression "pression atmosphérique ";

- Essaie de faire une phrase en français avec le mot « oxygène » ;

- Explique ce que veut dire le mot « oxygène».

Seules ces quatre dernières consignes figuraient dans le test des classes témoins.

Les scores obtenus par les élèves des classes immersives sont repris dans la Figure 3 :

Les scores sont globalement beaucoup plus faibles que ceux du graphique 1, ce qui semble logique étant donné qu'il ne s'agit plus ici de termes généraux, mais de termes reliés à des concepts scientifiques. La 
Articulation langue 1- langue 2 dans le répertoire langagier

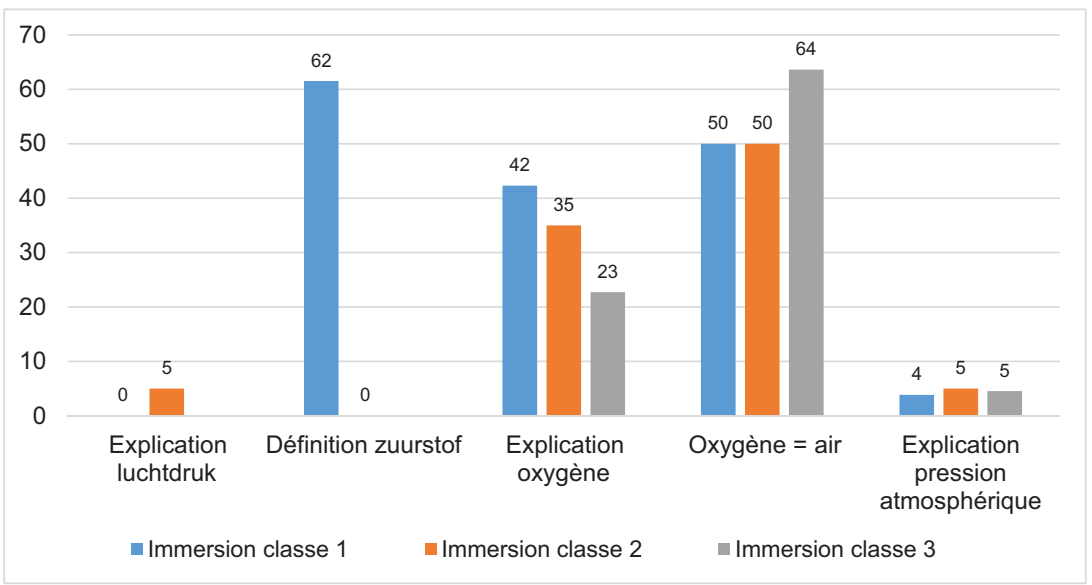

Figure 3 : Savoirs lexicaux initiaux 2 (groupes en immersion)

plupart des élèves n'ont pu apporter aucune explication ou exploitation discursive de luchtdruk (" pression de l'air ») et pression atmosphérique. A ce niveau, une précision s'impose. Lorsque nous avons élaboré les prétests, nous avons fait le choix, en français, d'utiliser le syntagme "pression atmosphérique ", car, après une recherche documentaire, il est apparu que c'était l'expression utilisée dans les programmes de sciences en communauté française de Belgique. Ce terme est cependant beaucoup moins transparent que "pression de l'air » (qui est la traduction littérale de luchtdruk) et il est par ailleurs relié au champ sémantique de l'astronomie. Il y a là un biais que nous avons tenté de contourner au moment des entretiens en utilisant successivement les deux syntagmes, malgré leurs nuances sémantiques.

Seuls des élèves de la classe $1(62 \%)$ parviennent à définir le terme zuurstof (« oxygène »). Dans les autres classes, les élèves ne répondent pas ou apportent une réponse erronée. ${ }^{6}$

Le terme " oxygène » est défini par 23 à $42 \%$ des élèves de chaque classe. Nous avons considéré comme correctes des définitions du type " quelque chose qui nous sert pour respirer ». Nous avons jugé utile d'indiquer dans le graphique le pourcentage d'élèves indiquant que l'oxygène est de l'air (au moins la moitié des élèves de chaque classe).

La Figure 4 indique les scores des élèves des classes témoins.

Il indique un pourcentage relativement faible d'élèves parvenant à expliquer le terme oxygène. Ce pourcentage devient nul pour l'expression "pression atmosphérique », sauf dans la classe 3.

En résumé, les élèves des trois classes immersives abordent leur séance sur l'air avec des savoirs lexicaux spécialisés faibles en 


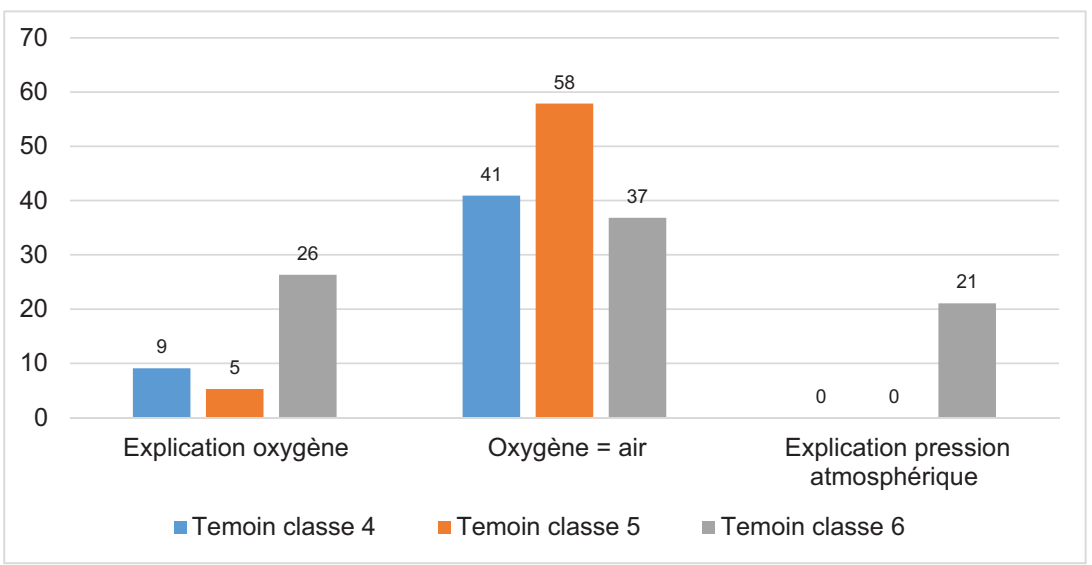

Figure 4 : Savoirs lexicaux initiaux 2 (groupes témoins)

néerlandais ainsi que des savoirs lexicaux généraux ne leur permettant pas nécessairement d'accéder de manière immédiate au sens des constituants notionnels des concepts centraux de la leçon : environ un tiers des élèves ne sont pas parvenus à identifier les items lucht (« air ») et bewegen («bouger»). Précisons que lucht signifie également ciel. Quant au verbe drukken (" pousser»), il est identifié par un relativement faible pourcentage d'élèves. Stilstaan ( « rester immobile»), en revanche, est identifié par un grand nombre d'élèves.

Comparativement, les élèves des classes témoins abordent leur séance sur l'air avec des savoirs lexicaux généraux plus solides, relativement aux termes qui nous ont semblé indispensables pour l'accès au sens, mais leur connaissance des éléments lexico-conceptuels spécialisés en français semble aussi faible que celle des élèves des classes immersives en néerlandais.

Nous allons maintenant voir sur quelles ressources lexico-conceptuelles les élèves s'appuient à l'issue de la séance. Nous allons envisager successivement deux sous-ensembles conceptuels en relation avec la construction conceptuelle de l'air :

- La notion de pression exercée par l'air ;

- Les catégorisations de l'air.

b) ressources lexico-conceptuelles des élèves à l'issue de la leçon : la pression exercée par l'air

Ce sous-ensemble conceptuel est principalement constitué, sur le plan sémantique, de la caractéristique « l'air exerce une 
pression partout », reliée en français sur le plan lexical aux syntagmes " pression » / "pression de l'air » / "pression atmosphérique » et aux verbes exprimant une action de pression.

Nous avons cherché, en analysant les entretiens en français, des indices concernant l'utilisation du lexique spécifique et les liens qui sont faits entre le lexique et les éléments sémantiques constitutifs du sous-ensemble conceptuel envisagé. Nous avons commencé par analyser les données tirées des entretiens réalisés avec les élèves des classes immersives, puis nous les avons systématiquement comparées à celles des entretiens réalisés avec les élèves des classes témoins.

Comment les élèves des classes immersives expriment-ils leur conceptualisation de la notion de pression ? À ce niveau, nous nous sommes tout d'abord intéressés aux verbes utilisés pour exprimer la pression. Dans les classes immersives, 86 à $100 \%$ des élèves utilisent spontanément un verbe exprimant la pression lorsqu'ils décrivent ou expliquent les expériences réalisées, ce verbe étant majoritairement le verbe " pousser », utilisé sous une forme intransitive (exemple : « l'air pousse ») ou transitive (exemple : " l'air pousse l'eau ») 21 à $27 \%$ des élèves utilisent également ou uniquement un autre verbe (appuyer, forcer, retenir, compresser, garder, coller, taper, tenir, bloquer, repousser). Les résultats sont beaucoup moins homogènes dans les classes témoins : le pourcentage d'utilisation spontanée d'un verbe exprimant la pression pour décrire ou expliquer les expériences est respectivement de $52 \%, 90 \%$ et $100 \%$, le verbe majoritairement utilisé étant à nouveau le verbe " pousser ». Les figures 5 et 6 reprennent l'ensemble des résultats.

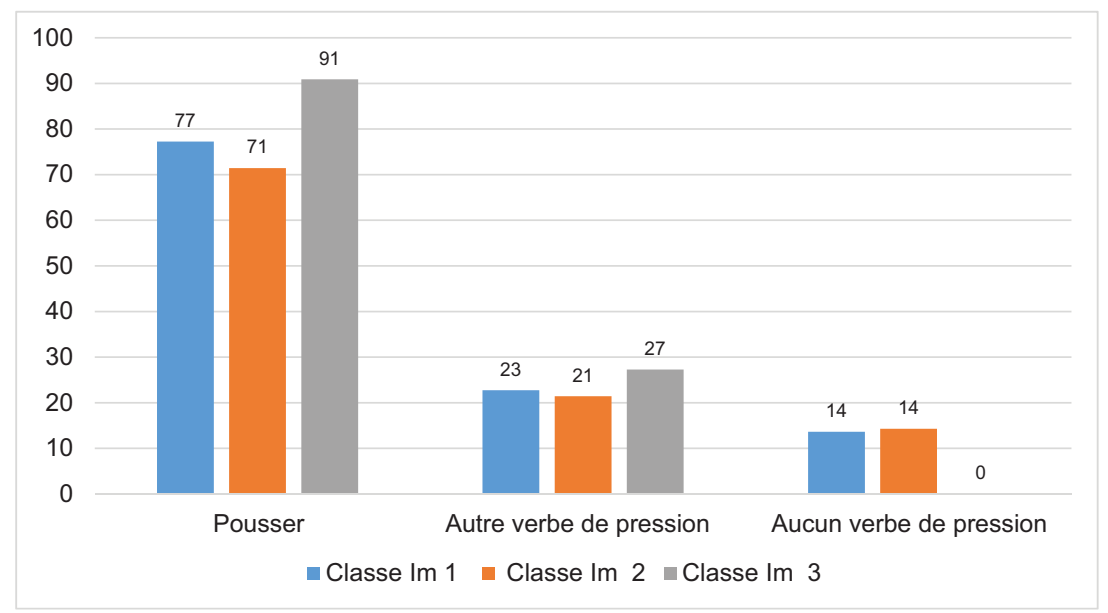

Figure 5 : Verbes d'action utilisés (groupes en immersion) 


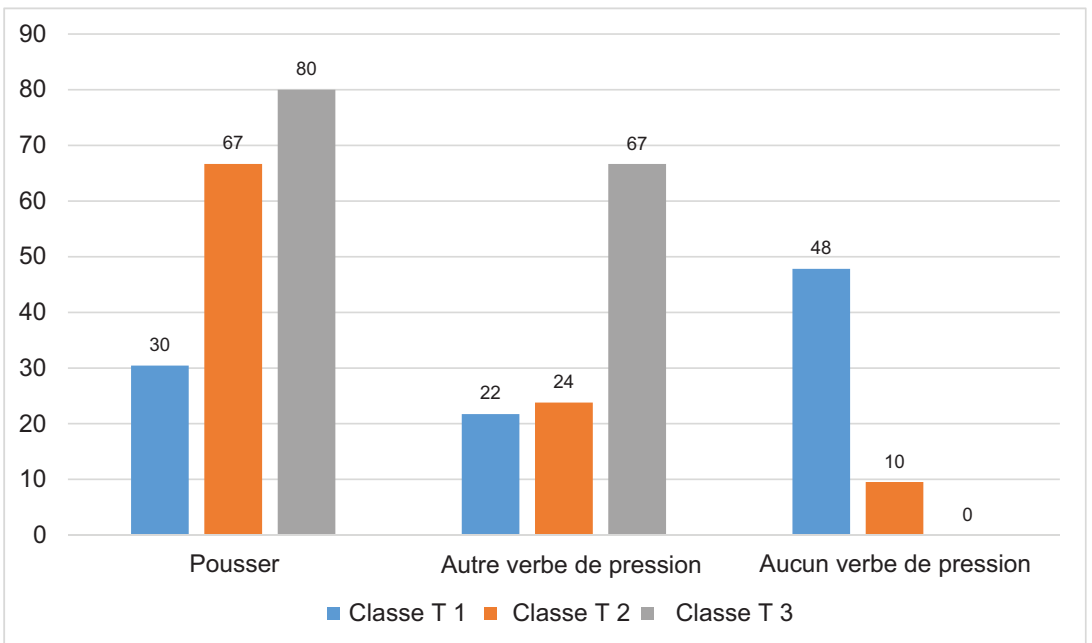

Figure 6 : Verbes d'action utilisés (groupes témoins)

Nous avons ensuite exploré les manières dont les élèves exprimaient, de manière plus générale, le fait que l'air exerce une pression. Il ne s'agissait plus pour nous de chercher à savoir, comme pour le premier point, si les élèves exprimaient une action pouvant relever de la description ou de l'explication d'une expérience donnée, mais bien de rechercher des marques de généralisation indiquant que les élèves avaient atteint un certain degré dans la construction du concept de pression de l'air comme facteur explicatif d'un ensemble de phénomènes. Ainsi, un énoncé tel que " $\mathrm{y}$ avait pas d'air au-dessus pour pousser l'eau » (M., classe Im 1) n'a pas été retenu, contrairement à " qu'est-ce que la maîtresse voulait montrer ? bah que l'air pousse quoi / il tombe sur nous » (L., classe $\operatorname{Im} 3$ ), qui envisage un espace de validité plus large au rôle joué par l'air. Nous avons trouvé peu d'occurrences spontanées de ces marques de généralisation (une dizaine au total dans les trois classes immersives, pratiquement aucune dans les classes témoins). Elles sont survenues soit dans l'introduction de l'entretien (B. classe Im 2 : « on a appris des choses sur l'air / que l'air pouvait pousser tout plein de choses »), soit lors au cours d'explications, durant lesquels elles prenaient une forme d'évidence $(\mathrm{M}$, classe $\operatorname{Im} 2$ : «vu que l'air il pousse tout partout. . .»).

Dans les classes immersives, environ un tiers des élèves utilisent spontanément, au moins une fois durant l'entretien, les termes " pression », "pression de l'air » ou " pression atmosphérique ». Interrogés sur la source de leurs savoirs, les élèves évoquent des éléments divers, 
qui se complètent probablement chez certains d'entre eux : lectures, émissions de télévision, informations apportées par les parents ou des membres de la fratrie, etc. Les pré-tests distribués aux élèves une semaine avant la séance consacrée aux expériences ont également mis les élèves en contact avec ces termes. On ne peut pas non plus exclure la transmission d'informations d'un élève à l'autre entre les entretiens. Ce point semble particulièrement clair pour la classe $\operatorname{Im} 3$, dont plus de la moitié des élèves évoquent la pression atmosphérique en réponse à la question initiale de l'enquêteur : " qu'est-ce que vous avez fait en classe ce matin ? ». Enfin, certains élèves semblent opérer une traduction à partir de luchtdruk, à l'instar de l'exemple suivant : « on a appris sur l'air euh qui pousse » (N, classe Im 1$)$.

De manière assez prévisible, le fait d'utiliser le terme "pression " ou une expression contenant ce terme ne constitue pas un indicateur de l'intériorisation par les élèves des contenus notionnels du concept. On observe en effet, aussi bien dans les classes immersives que dans les classes témoins, des utilisations de ces termes soit très approximatives soit indiquant clairement une confusion avec un autre terme, comme on le voit dans les exemples suivants :

$\mathrm{N}^{7}$., classe $\operatorname{Im} 1$

I62 : quand la bougie s'est éteinte y a eu trop de pression et l'eau elle est remontée dans le verre //

E63 : pourquoi y avait trop de pression? /

I64 : parce que l'eau chaude et l'eau froide quand ça se mélange ça fait une sorte de fumée /

M., classe T3

I32 : en fait euh on allumait le sèche-cheveux / et euh: / on / on l'allumait / et euh aussi comme on / on: / place la balle de pingpong elle reste euh bien préci / elle / / elle tient parce que / déjà y a de l'ai / euh y a de l'air euh de la pression partout là où on est et: / plus (+) euh le / la pression du sè / de l'air du sèche-cheveux et ben elle tient en équilibre /

A., classe T3

E11 : un peu? / et sinon le reste alors / enfin qu'est-ce qui peut expliquer ça encore ? / y avait / est-ce qu'y avait quelque chose qui poussait le jus d'orange ou pas? / 


\section{Babault et Markey}

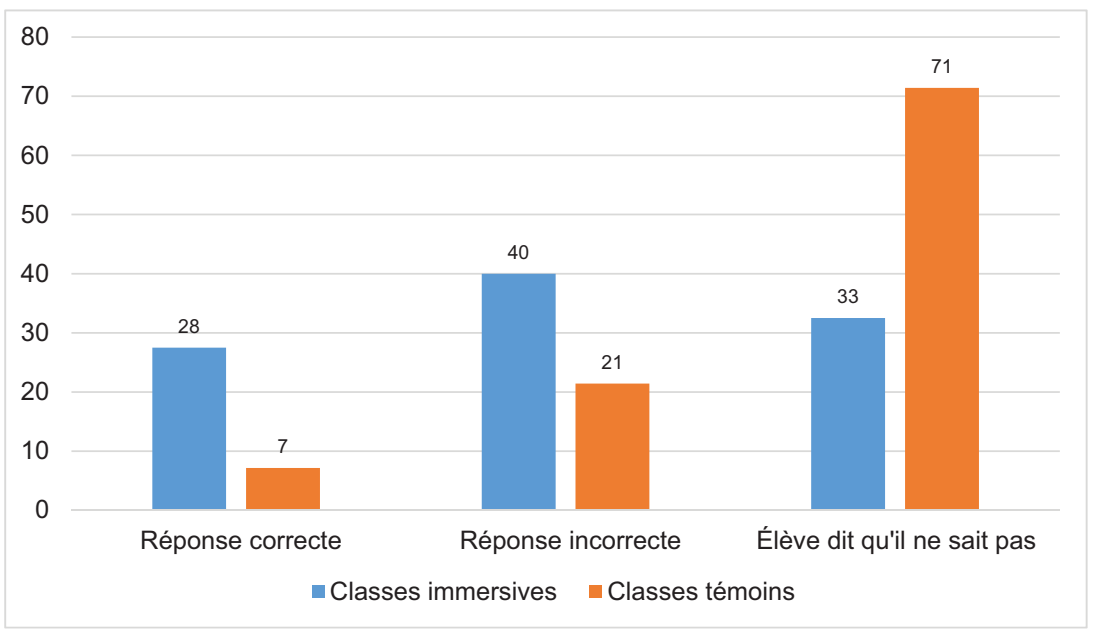

Figure 7 : Définition de pression de l'air / pression atmosphérique

I12 : bah: oui quand même la pression / qui faisait monter le jus d'orange dans le verre /

E13 : la pression euh / de du verre ou:: ? / ou / quelle pression ? /

I14 : ben la pression autour euh: qui: / ben qui est: / l'oxygène quoi /

Enfin, analysons maintenant les réponses apportées par les élèves à la demande de définition faite à la fin de l'entretien : " que veut dire pression atmosphérique ? / que veut dire pression de l'air ? ». La Figure 7 synthétise, pour les classes immersives et les classes témoins, le type de réponse apportée par les élèves : réponse correcte, réponse incorrecte, élève déclarant explicitement qu'il ne sait pas.

Nous avons retenu comme réponse correcte des énoncés tels que :

c'est quand l'air pousse quelque chose (R., classe Im 1)

ça veut dire que ça pousse tout partout (M. cl Im 2)

c'est la poussée de l'air / de tous / sur tous les côtés (U., classe T 1)

comme pression presse / y nous avait dit ça // ben pression c'est comme euh l'air euh / l'air euh qui presse un truc comme ça je sais pas (N., classe T 3) 
que l'air elle pousse / elle pousse sur nous (An., classe T 3)

l'air il pousse sur quelque chose (A. , classe T 3)

Nous avons également retenu comme réponse correcte les réponses qui faisaient référence à la pression atmosphérique de manière plus générale :

c'est ce qui fait qu'on reste sur terre / qu'on s'envole pas (L., classe $\operatorname{Im} 3$ )

Nous avons considéré comme incorrectes des réponses telles que les suivantes :

$c^{\prime}$ est quand il n'y a plus d'air (R, classe $\left.\operatorname{Im} 1\right)$

c'est l'air qui bouge (L., classe Im 1)

c'est rejeter de l'air (T., classe Im 1)

la pression ça veut dire que comme par exemple avec cette bougie / quand elle a eu besoin d'air elle a utilisé la pression pour prendre de l'air en bas (L., classe Im 1)

il y a quelque chose qui pousse l'air vers quelque chose (S., classe Im 1)

c'est l'air qui pousse pas très bien (B., classe Im 2)

c'est le vent l'oxygène tout ce qui est autour quoi (J., classe Im 2)

c'est l'air qui se bloque / donc euh l'air poussé (M., classe Im 3)

c'est la pression dans l'air (A., classe T 1)

la pression elle pousse (R., classe T 2)

la pression c'est à peu près l'air mais plus fort et ça pousse (H., classe T 2)

ça veut dire que l'air elle est forte (S., classe T 2)

c'est la pression qui pousse sur l'atmosphère (M., classe T 3)

Le fait de ne pas avoir reçu d'explications en français ne semble donc pas nécessairement avoir désavantagé les élèves des classes immersives. Précisons que cette tâche de définition ne correspondait pas pour les élèves à la répétition d'une définition fournie en classe (ce qui 
n'a pas été explicitement le cas dans les six classes concernées) mais plutôt à un travail d'induction à partir du thème de la leçon et des éléments de caractérisation découverts en classe. On comprend d'ailleurs, en lisant les occurrences considérées comme correctes, que les élèves se sont appuyés sur différentes stratégies pour définir les termes à la demande de l'enquêteur :

- Reprise mot pour mot du discours de l'enseignant (« ça veut dire que ça pousse tout partout ») ;

- Reformulation («c'est quand l'air pousse quelque chose») ;

- Appui sur la morphologie des termes ("pression presse») ;

- Appui probable sur un savoir développé avant la leçon (« c'est ce qui fait qu'on reste sur terre »).

Nous avons cherché à éclairer cette analyse par la prise en compte des réponses apportées à la demande équivalente en néerlandais à propos du terme luchtdruk. Le premier constat est celui d'une faible corrélation entre le fait d'apporter une réponse correcte, non correcte ou pas de réponse en français ou en néerlandais (coefficient de corrélation : 0,28). Autrement dit, ce n'est pas parce qu'un élève sait répondre à la consigne de définition en néerlandais qu'il saura le faire également en français, et inversement. On pourtant retrouve les mêmes types de stratégies, avec cependant une proportion très forte d'analyse lexicale, facilitée en néerlandais par la forme du syntagme dont la définition est demandée ("luchtdruk is lucht die drukt ", trad. : la pression de l'air c'est de l'air qui presse), ainsi qu'un recours à la traduction en français ("luchtdruk is euh pression atmosphérique »), sans que l'on puisse savoir quels contenus conceptuels l'élève met derrière le terme en français.

\section{c) ressources lexico-conceptuelles des élèves à l'issue de la leçon : les catégorisations de l'air}

Dans leur leçon, les enseignants des six classes ont insisté fortement sur un deuxième sous-ensemble conceptuel : celui de la catégorisation de l'air. En effet, dans la construction du concept « air », tel que présenté par les enseignants, entrent en ligne de compte des oppositions binaires liées à la température et au mouvement, entraînant des différences de force : air froid vs air chaud et air immobile vs air mobile, chacun des premiers termes de l'opposition présentant une force supérieure à l'autre. Nous allons maintenant analyser comment les élèves gèrent ces données sur le plan linguistique et conceptuel.

La Figure 8 reprend, pour les classes immersives et les classes témoins, la présence ou non de ces oppositions binaires dans les productions en français. Les assez faibles occurrences de certaines catégories 
Articulation langue 1- langue 2 dans le répertoire langagier

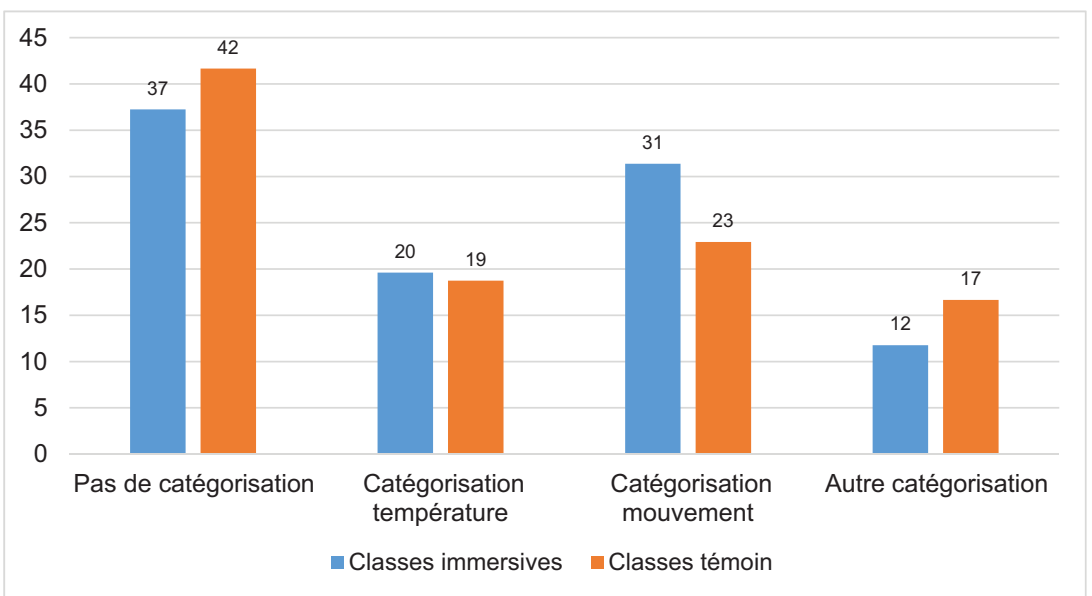

Figure 8 : Catégorisation de l'air dans les productions spontanées

nous ont conduits à comptabiliser les occurrences suivant la distinction classes immersives / classes témoins et non pour chaque classe.

On n'observe pas de différences extrêmement marquées entre les classes immersives et les classes témoins. De manière globale, plus d'un tiers des élèves n'énoncent pas de catégorisation, les catégorisations liées à la température ou au mouvement sont citées par un tiers des groupes (l'une n'excluant pas l'autre), tandis que 12 à $17 \%$ des élèves font référence à d'autres formes de catégorisation, reliées à des considérations spatiales (air intérieur / air extérieur ; air du dessus / air du dessous). Nous avons été particulièrement intéressés par les dénominations utilisées par les élèves pour exprimer ces catégorisations. Sur ce plan, les classes immersives se démarquent fortement des classes témoins, en particulier en ce qui concerne la catégorisation liée au mouvement. Si les élèves des groupes témoins utilisent exclusivement du type " air qui bouge / air qui ne bouge pas ", les productions des élèves des classes immersives présentent une diversité beaucoup plus importante. "L'air qui bouge » est ainsi opposé aux dénominations suivantes :

- Air qui ne bouge pas

- Air stable

- Air droit

- Air plat

- Air calme

- Air silencieux

- Air tranquille 
C'est manifestement l'adjectif stilstaand ("immobile») qui est à la source de ces dénominations. Pour mémoire, le verbe stilstaan (« rester immobile ») avait été reconnu par une majorité d'élèves des classes immersives lors des prétests. Il ne s'agit cependant plus ici nécessairement de reconnaissance et de compréhension du terme, mais de transfert de la L2 vers la L1. L'utilisation des dénominations autres que " air qui ne bouge pas » peut être reliée à des focalisations sémantiques réalisées par les élèves à partir de l'un ou l'autre des constituants lexicaux du syntagme néerlandais. Staan (" être debout») est peut-être à l'origine de la dénomination air droit . Les autres dénominations semblent directement en lien avec les différentes acceptions de l'adjectif stil : calme, tranquille, silencieux, etc. Les élèves qui ont utilisé ces dénominations en français semblent s'être appuyés sur leur analyse sémantique du terme néerlandais plutôt que sur la prise en compte d'une relation sémantique entre les termes utilisés en français.

On retrouve, dans une certaine mesure, cette diversité lexicale en ce qui concerne le terme générique utilisé pour air. Si la majorité des élèves des classes immersives et des classes témoins utilisent effectivement le terme air, un pourcentage non négligeable (10\% des classes témoins et $18 \%$ des classes immersives) utilise un autre terme : oxygène, pression atmosphérique ou vent (ce dernier terme apparaissant uniquement dans les productions d'élèves des classes immersives). Des exemples de ces utilisations sont présentés dans les extraits ci-dessous :

c'est parce que autour il y avait de l'oxygène / je sais pas comment on dit en français (T., classe Im 1)

on devait remplir un verre avec de l'eau et mettre une cass une carte postale euh:: du côté de l'image / et puis appuyer un peu pour dire que l'oxygène elle part / puis après retourner (A., classe T 1)

on a aussi appris que la pression atmosphérique chaude elle a moins d'effet que celle qui est froide (O., classe $\operatorname{Im} 3$ )

parce que avec la bougie qui s'était éteint / ah ben l'eau elle a remonté parce que / il y avait de l'air chaud / de la pression atmosphérique chaude / (. . .) la pression atmosphérique chaque est moins forte que celle euh froide (M., classe T 1)

parce que le vent froid il était dans l'assiette et le vent chaud il était dans le verre (R., classe Im1) 
Ces exemples semblent indiquer un traitement lexical analogue des termes concernés par les élèves des classes immersives et des classes témoins. Par ailleurs, la proportion de ce type d'occurrences, même si elle est plus importante dans les classes immersives, n'est pas suffisamment éloignée pour pouvoir conclure à des réactions différentes entre les deux groupes. Seule peut finalement être retenue de manière nette la différence que nous avons observée au niveau de l'opposition mobile / immobile.

\section{Conclusion}

Nous posions dans l'introduction de cet article la double question de la compétence bilingue et de l'existence de passerelles entre la L2 et la L1 dans la construction lexico-conceptuelle opérée par les élèves des programmes immersifs.

Commençons par noter le fait que, en dépit de savoirs lexicaux initiaux plus réduits que ceux de leurs camarades des classes témoins, les élèves des classes immersives montrent à l'issue de la leçon des traces de construction conceptuelle relativement comparables.

Sur le plan lexical, les productions des élèves indiquent plus ou moins de convergence en fonction des items pris en compte. Pour certains items, on observe chez les élèves des classes immersives un recours à une plus grande variété lexicale en français que les élèves des classes témoins, cette variété pouvant être reliée aussi bien à une mise en œuvre élargie des éléments français du répertoire lexical (comme pour les verbes décrivant la pression) qu'à une recherche d'indices au niveau de la forme des éléments néerlandais de ce répertoire (comme cela a pu être constaté au niveau de la catégorisation).

Notons enfin la présence d'une grande hétérogénéité dans les comportements discursifs et métadiscursifs des élèves des classes immersives : si certains semblent avoir acquis une souplesse lexicale leur permettant de s'appuyer sur l'ensemble de leurs ressources pour réaliser les tâches demandées dans une langue ou l'autre, d'autres mettent explicitement en avant une frontière psycholinguistique susceptible de fonctionner aussi bien dans les discours en français que dans ceux en néerlandais : « je sais le dire en. . . mais pas en. .. ». Les données dont nous disposons ne nous permettent pas de déterminer si cette frontière qui fonctionne sur le plan discursif présente des contours aussi nets sur le plan conceptuel.

La correspondance devrait être adressée à Sophie Babault, département des sciences du langage, université Lille 3, BP 60149, 59653 Villeneuve d'Ascq cedex, France. Courriel : sophie.babault@univ-lille3.fr. 


\section{Notes}

1 Mobärg (1997 : 206) : «L2 learners enter the language with a ready-made set of concepts ».

2 Nous avons d'ailleurs dû modifier l'échantillon en remplaçant une classe du groupe témoin dont le profil des élèves s'est révélé lors de l'expérimentation trop différent de celui des classes immersives.

3 Nous remercions les directeurs et enseignants de ces établissements pour leur accueil et la disponibilité dont ils ont fait preuve.

4 L'équipe était composée des deux rédacteurs de cet article et de quatre étudiantes belges néerlandophones qui ont été chargées de réaliser les entretiens en néerlandais : Scarlet Coopman, Annelies Hajima, Sien Kums et Lieselot Perneel.

Nous profitons de cet article pour remercier encore une fois chaleureusement ces quatre étudiantes dont nous avons apprécié autant les compétences que les qualités humaines!

5 D'autres items, tels que des prépositions spatiales et des quantitatifs, étaient également testés, mais nous ne les utiliserons pas dans cet article.

6 Parmi ces réponses erronées figurent notamment des tentatives intéressantes d'interprétation à partir du radical zuur (« acide »).

7 Conventions de transcription des entretiens : /=pause courte ; //=pause longue ; : = allongement de syllabe ; $\mathrm{I}=$ informateur ; $\mathrm{E}=$ enquêteur.

\section{Références}

Alanen, R., Jäppinen, A.-K. et Nikula, T. (2006). “But big is a funny word": a multiple perspective on concept formation in a foreign-language-mediated classroom. Journal of Applied Linguistics, 3(1), 69-90.

Astolfi, J.-P., Peterfalvi, B. et Vérin, A. (1998). Comment les enfants apprennent les sciences ? Paris: Retz.

Babault, S. (2008). Transferts de contenus scolaires de la L2 à la L1 en contexte d'immersion : mémoire bilingue et activités de catégorisation. Dans K. Haataja (dir.), Linguistic diversity through Integration, Innovation and Exchange. (pp. 99116). Tampere: Juvenes Print.

Babault, S. et Markey, M. (2011). Des savoirs disciplinaires bilingues chez les élèves en immersion? Dans L. Beheydt. et P. Hiligsmann (dirs.), Met immersie aan de slag/Au travail, en immersion. (pp. 93-109) Louvain: Presses Universitaires de Louvain.

Brossard, M. et Fijalkow, J. (dirs.). (2002). Apprendre à l'école : perspectives piagétiennes et vygotskiennes. Bordeaux: Presses Universitaires de Bordeaux.

Cummins, J. (1980). The cross-lingual dimensions of language proficiency: implications for bilingual education and the optimal age issue. TESOL Quarterly, 14(2), 175-187. http://dx.doi.org/10.2307/3586312.

de Groot, A. (1993). Word-type effects in bilingual processing tasks. Support for a mixed representational system. Dans R. Schreuder et B. Weltens (dirs.), The 
bilingual lexicon (pp. 27-51). Amsterdam: John Benjamins. http:/ / dx.doi.org/ 10.1075/sibil.6.04gro.

Gajo, L. (2007). Linguistic knowledge and subject knowledge: How does bilingualism contribute to subject development? International Journal of Bilingual Education and Bilingualism, 10(5), 563-581.

Gajo, L. et Serra, C. (2000). Enseignement bilingue et apprentissage des mathématiques. Études de linguistique appliquée, 120, 497-508.

Genesee, F. (1984). On Cummins' theoretical framework. Dans C. Riviera (dir.), Language proficiency and academic achievement (pp. 20-27). Clevedon: Multilingual Matters.

Giordan, A. et de Vecchi, G. (1994). Les origines du savoir: des conceptions des apprenants aux concepts scientifiques. Lausanne: Delachaux \& Niestlé.

Jaubert M. (2007). Langage et construction de connaissances à l'école : un exemple en sciences. Bordeaux: Presses universitaires de Bordeaux.

Lüdi, G. (1999). Alternance des langues et acquisition d'une langue seconde. Cahiers du français contemporain, 5, 25-52.

Malakoff, M. (1988). The effect of language of instruction on reasoning in bilingual children. Applied Psycholinguistics, 9(1), 17-38. http:/ /dx.doi.org/10.1017/ S0142716400000436.

Mobärg, M. (1997). Acquiring, teaching and testing vocabulary. International Journal of Applied Linguistics, 7(2), 201-222. http:/ / dx.doi.org/10.1111/j.14734192.1997.tb00115.x.

Nation, I. S .P. (2001). Learning vocabulary in another language. Cambridge: Cambridge University Press. http:/ /dx.doi.org/10.1017/CBO9781139524759.

Noyau, C. (2003). Apprentissages disciplinaires et langagiers dans la scolarisation en français langue seconde : repérages dans le temps en histoire au primaire.

Dans J. P. Bernié, M. Jaubert et M. Rebière (dirs.), Construction des connaissances et langage dans les disciplines d'enseignement (Actes sur CD-Rom), CIRFEMDAEST, Université Victor Segalen-Bordeaux II (pp. 1-10).

Noyau, C. (2007). L'enseignement de disciplines non linguistiques en FLS au primaire en Afrique de l'ouest : les sciences d 'observation à travers les manuels et les activités de classe. TREMA, 28(28), 49-61. http:/ /dx.doi.org/ $10.4000 /$ trema. 282.

Noyau C. et Vellard D. (2004). « Construction de connaissances mathématiques dans la scolarisation en français langue seconde ». Cahiers $d u$ français contemporain 9 ( Pratiques et représentations langagières dans la construction et la transmission des connaissances »). 57-76.

Robinson, P. (dir.) (2001). Cognition and second language instruction. Cambridge: Cambridge University Press. http:/ /dx.doi.org/10.1017/CBO9781139524780.

Schneeberger, P. et Vérin, A. (2009). Développer des pratiques d'oral et d'écrit en science : quels enjeux pour les apprentissages à l'école? Paris: INRP.

Stohler U. (2006). The acquisition of knowledge in bilingual learning : an empirical study on the role of learning in content learning. Vienna English working papers (special issue : current research on CLIL). 15(3), 41-46. 


\section{\Babault et Markey}

Sylvén, L. K. (2010). Teaching in English or English teaching? On the effects of content and language integrated learning on Swedish learners' incidental vocabulary acquisition. Göteborg: Department of English, Göteborg University.

Van de Craen, P., Surmont, J., Mondt, K. et Ceuleers, E. (2012). Twelve years of CLIL practice in multilingual Belgium. Dans G. Egger et C. Lechneer (dirs.), Primary CLIL around Europe: Learning in two languages in primary education (pp. 81-97). Marburg: Tectum.

Verhoeven, L.T. (1994). Transfer in Bilingual Development: The Linguistic Interdependence Hypothesis revisited. Language Learning, 44(3) 381-415.

Vigner G. (2001). Enseigner le français comme langue seconde. Paris: CLE international.

Vygotski, L. (1934, trad. française 1985). Pensée et langage. Paris: La Dispute. 